\title{
EFECTO DEL RALEO SOBRE LAS PROPIEDADES ANATÓMICAS DE LA MADERA DE Pinus taeda
}

\section{EFFECT OF THINNING ON THE ANATOMICAL PROPERTIES OF Pinus taeda WOOD}

\author{
Rosa Ángela Winck ${ }^{1, \AA}$, Hugo Enrique Fassola ${ }^{1}$, María Cristina Area $^{2}$
}

\begin{abstract}
RESUMEN
El raleo tiene implicancias económicas y técnicas para producción forestal y para la actividad industrial. El objetivo del estudio fue determinar la influencia de la intensidad de raleo sobre las propiedades anatómicas de la madera. Se trabajó con muestras tomadas a 1,30 m de altura, de 24 árboles proveniente de un ensayo de raleo en Pinus taeda de 20 años de edad, intervenido a los 3 años, con remoción del $0 \%, 50 \%, 75 \%$ y $87,5 \%$ de las plantas de la densidad inicial de plantación (1960 árboles/ ha). Se midieron diámetro a la altura de pecho, altura total, diámetro en la base de la copa viva y altura a la base de la copa viva, longitud de traqueidas, espesor de pared celular y ángulo microfibrilar. La longitud de traqueidas fue mayor en el tratamiento con $87,5 \%$ de raleo. Los tratamientos con $0 \%$ y $50 \%$ de raleo favorecieron la obtención de mayores valores de espesor de pared y menores valores de ángulo microfibrilar. Se concluyó que esta práctica silvícola altera a las propiedades anatómicas de la madera de Pinus taeda y que la longitud de traqueidas está fuertemente relacionada con el tamaño del árbol y con la longitud de la copa verde.
\end{abstract}

Palabras clave: Ángulo microfibrilar, espesor de pared celular, intensidad de raleo, longitud de traqueidas.

\begin{abstract}
Thinning has technical and economic implications for both, forest production and industrial activity. The aim of this study was to determine the effect of thinning intensity on the anatomical properties of pine wood. Samples were taken at $1.30 \mathrm{~m}$ height, from 24 Pinus taeda trees of 20 years old, coming from a thinning treatment. The plantation was intervened at 3 years, with removal of $0 \%, 50 \%, 75 \%$ and $87,5 \%$ of the trees from the initial density (1960 trees/ha). The evaluated variables were: diameter at breast height, total height, diameter on the base of the live crown, height at base of live crown, tracheid length, cell wall thickness and microfibril angle. The tracheid length was higher in $87.5 \%$ thinning treatment. Treatments with $0 \%$ and $50 \%$ thinning have favored the obtaining of higher values of cell wall thickness and lower values of microfibril angle. Results suggest that this silvicultural practice alters the anatomical properties of Pinus taeda wood and that tracheid length is strongly related to tree size and length of live crown.
\end{abstract}

Keywords: Cell wall thickness, microfibril angle, thinning intensity, tracheid length.

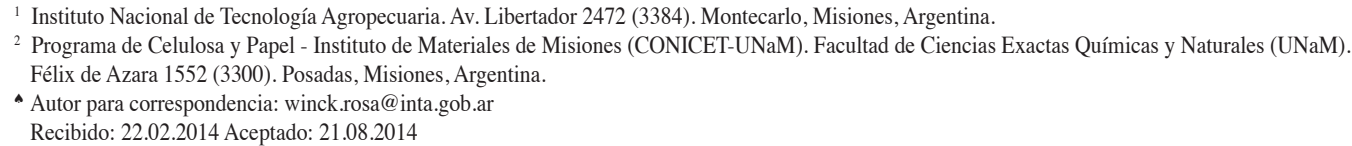




\section{INTRODUCCIÓN}

Se han desarrollado en la Argentina, a partir de la década de 1990, estudios forestales relacionados a aspectos biológicos y biométricos con una visión sistémica, lo que permite contar con información estructurada para las principales especies mediante la cual es factible cuantificar las existencias actuales e inclusive efectuar prognosis de la evolución de los rodales bajo determinadas circunstancias. El uso de la información existente es relevante en la planificación de los bosques a mediano y largo plazo herramienta a la que los silvicultores han recurrido para lograr una mayor diversificación de productos y para aumentar el valor del patrimonio forestal. En la provincia de Misiones, situada al NE de la Argentina, se encuentra la mayor superficie plantada con pinos del país, los cuales han sido sometidos a años de mejoramiento genético. Sin embargo, existe un vacío en el conocimiento de sus características anatómicas y tampoco se han encontrado trabajos acerca del efecto del raleo sobre las propiedades de la madera. En este sentido, el Pinus taeda constituye una de las especies de mayor importancia, dada la extensa superficie implantada, su alta tasa de crecimiento y su potencialidad de uso tanto para la construcción como carpintería en general.

El conocimiento de los factores que influyen en la calidad de la madera, es trascendente para mejorar el valor de las plantaciones (Sarén et al. 2004). Para mejorar la competitividad de las empresas, se debe apuntar a la obtención de madera de calidad, lo que implica la incorporación de nuevas características que sean representativas de la aptitud de la madera para uso sólido. Las características anatómicas están íntimamente relacionadas con las propiedades de la madera y por lo tanto, con su procesamiento tecnológico. Por este motivo la longitud de traqueidas, el espesor de la pared celular y el ángulo microfibrilar son consideradas de gran importancia.

El ángulo microfibrilar (AMF) tiene un efecto significativo sobre las propiedades mecánicas (Hein y Lima 2012, Hein et al. 2013) y la estabilidad dimensional de la madera, y como tal es un parámetro indicador de la calidad de los productos de madera maciza (Macdonald y Hubert 2002). Las propiedades de la madera mejoran en la medida que el AMF disminuye. La orientación de las fibrillas en la capa S2 de la pared secundaria de las traqueidas determina en gran medida las propiedades de la madera (Cave y Walker, 1994; Walker y Butterfield 1995), sobre todo la resistencia y la contracción longitudinal (Ying et al. 1994, Walker y Butterfield, 1995, Donaldson 1996), constituyendo un factor crítico en sus propiedades mecánicas (Megraw 1985, Bendtsen y Senft, 1986). Pese a la influencia del AMF en las propiedades de la madera, las investigaciones son limitadas debido a lo dificultoso que resulta su medición. La orientación de las puntuaciones y de los campos de cruzamiento en la pared de las traqueidas ha sido empleado por varios autores para medir el AMF ya que es indicativa de la alineación de las microfibrillas.

El objetivo del presente trabajo fue determinar el efecto de la intensidad de raleo sobre la longitud de traqueidas, el espesor de pared y el ángulo microfibrilar de la madera de Pinus taeda de 20 años de edad. 


\section{MATERIALES Y MÉTODOS}

Se estudiaron árboles provenientes de un ensayo de poda y raleo de Pinus taeda ubicado en el lote 13A, propiedad de la Empresa APSA S.A., en Puerto Bossetti, Departamento Iguazú, provincia de Misiones, Argentina (Figura 1). Geográficamente se ubica a los $26^{\circ} 18^{\prime} \mathrm{S}$ y $55^{\circ} 38^{\prime} \mathrm{O}$. La plantación fue realizada en 1992 con una densidad inicial de 1960 plantas por hectárea, en 1995 se instaló el ensayo de raleo. Se adoptó el diseño sistemático clinal considerando una faja sin raleo ( $0 \%$ y fajas con un $50 \%, 75 \%$ y $87,5 \%$ de la densidad original (1960 plantas/ha). Cada faja constó de una bordura perimetral. El ensayo quedó constituido por cuatro tratamientos: $\mathrm{T} 0=0 \%, \mathrm{~T} 1=50 \%, \mathrm{~T} 2=75 \%$ y T3 $=87 \%$, intensidades de raleo con respecto a la densidad original, con 1960, 980, 490 y 245 plantas por hectárea respectivamente. Para este estudio se consideró únicamente el tratamiento de raleo.

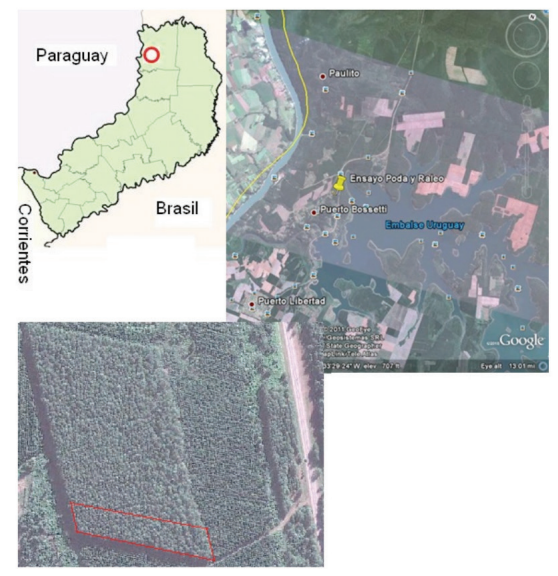

Figura 1. Ubicación del ensayo de raleo de Pinus taeda.

El suelo se clasifica como "Kandiudult” (Soil Survey Staff 1975). Se caracteriza por un desarrollo en profundidad mayor a los $2 \mathrm{~m}$ con secuencia de horizontes y capas A-Bt-C, rojo, libre de pedregosidad y fragmentos gruesos, permeabilidad moderada, bien drenado y relativamente insaturado.

El clima de la región se caracteriza por una temperatura media anual de $21^{\circ} \mathrm{C}$ y precipitaciones de alrededor de $2000 \mathrm{~mm}$ anuales, con régimen isohigro (INTA 1990, Servicio Meteorológico Nacional 2008).

\section{Selección de los árboles}

Se seleccionaron 6 árboles de cada tratamiento ( 24 en total), de 20 años de edad sin condición de borde, con buen estado sanitario, con fustes derechos, sin bifurcaciones. Para la selección se tuvo en cuenta su posición fitosociológica: suprimidos, codominantes y dominantes, de acuerdo con Köhl et al. (2006), y se tomaron 2 ejemplares de cada estrato. Cada árbol fue correctamente identificado, midiéndose con cinta dendrométrica el diámetro a la altura de pecho $(1,30 \mathrm{~m})$ y el diámetro en la base de la copa viva, y con cinta métrica la altura total y la altura hasta la base de la copa viva del árbol. Se apearon, se trozaron y se extrajeron rodajas de $2,5 \mathrm{~cm}$ de espesor a 1,30 metros de altura en el fuste.

De cada rodaja se sacó un listón central de $2,5 \mathrm{~cm}$ de ancho, en el cual se marcaron probetas (bloques) de $2 \times 2 \times 2 \mathrm{~cm}$ de 3 posiciones en el radio, que se identificaron como: interno (A: bloque inmediato a la médula, que abarcó aproximadamente entre los anillos 0 y 3), medio (B: al 50\% del radio, que abarcó aproximadamente entre los anillos 3 y 9) y externo (C: el bloque adyacente a la corteza, que abarcó aproximadamente entre los anillos 10 y 20). De estos 3 bloques, se tomaron muestras en sentido paralelo a las fibras, las cuales se cortaron con un formón en forma de prismas (de tipo "palillos") de 2 a $4 \mathrm{~mm}$ de 
espesor y ancho y $20 \mathrm{~mm}$ de largo. Se realizó la impregnación de los prismas hirviéndolos en agua durante 24 horas (hasta lograr el ablandamiento del material). Se empleó la técnica de maceración de Nuñez y Pavlik (1999) con dióxido de cloro-ácido acético-carbonato de sodio. La técnica se basa en la generación de dióxido de cloro a partir de clorito de sodio para ablandar o disolver lignina de la lámina media hasta llegar al punto de separación de fibras (no se requiere sacar la totalidad de la lignina). El dióxido de cloro es gaseoso a temperatura ambiente tiene la ventaja de ser un agente selectivo y prácticamente no daña a los polisacáridos. La extensión del tratamiento con dióxido de cloro a temperatura ambiente depende de la especie. El tratamiento consistió en colocar los prismas en tubos de ensayos con suficiente cantidad de agua para cubrirlos completamente, se agregó el clorito en solución alcanzando una concentración final de 2-5\% como cloro activo. Se mezcló y se agregó $5 \mathrm{ml}$ de ácido acético concentrado con agitación continua. En unos instantes se observó la formación de dióxido de cloro que es un gas de color amarillo anaranjado. Se tapó el recipiente con papel de aluminio y se dejó bajo campana en laboratorio. El final del proceso se determinó empíricamente, hasta lograr el nivel necesario de deslignificación, es decir la separación de los elementos celulares. Para neutralizar se extrajeron los prismas y se colocaron a hervir en una solución de carbonato de sodio de 5-10\% por 15-20 minutos Luego se realizó el lavado con agua de red para extraer toda la solución. Las traqueidas se tiñeron con safranina al $1 \%$ y se colocaron en portaobjetos, agregando media gota de glicerina y cubriendo con cubre objetos.

Sobre el material macerado se determinó la longitud de traqueidas, espesor de pared y ángulo microfibrilar.

\section{Medición de caracteres anatómicos}

Para las observaciones y fotomicrografías se utilizó un Microscopio óptico marca Zeiss Modelo Axiolab, con cámara fotográfica marca Moticam 2000 con software para mediciones celulares incorporado.

El AMF se midió con la técnica de Huang et al. (1998) y Wang et al. (2001) a través de las puntuaciones, utilizando el software UTHSCSA "Image Tool” para Windows versión 3.0 (Wilcox et al. 2002) previamente calibrado. Para medir el espesor de pared y AMF se tomaron fotomicrografías de las traqueidas con un aumento de 1000X. En la fotomicrografía de cada traqueida se midieron tres ángulos microfibrilares.

Para medir la longitud de traqueidas se empleó un aumento de 40X. Las mediciones celulares se hicieron siguiendo las recomendaciones de las normas IAWA (Richter et al. 2004).

Se realizaron 50 mediciones de espesor de pared y longitud de traqueidas y 100 mediciones de ángulo microfibrilar por cada muestra, con un total de 3600 mediciones de espesor de pared y longitud de traqueidas y 7200 mediciones de AMF.

Se realizó el análisis de las variaciones de las propiedades anatómicas en 3 posiciones radiales del fuste, a una única altura $(1,30 \mathrm{~m})$. Las traqueidas de leño temprano y tardío se determinaron inicialmente a través de la distribución bimodal de las distribuciones de frecuencia y luego se validó experimentalmente en laboratorio.

Se aplicó un análisis de varianza a los efectos de evaluar las características de los árboles muestreados para los distintos tratamientos. 
Los datos de los caracteres anatómicos, por tratarse de datos longitudinales (medidas repetidas de un mismo sujeto) no cumplen con el supuesto de independencia, por lo tanto se analizaron con modelos lineales generalizados mixtos (ANCOVA). Para emplear estos modelos, se buscó la distribución (función de enlace "g") que mejor representaba los datos. Estas fueron la Gaussiana para AMF y la Gamma para espesor de pared y longitud de traqueidas.

Como fuente de variación se consideraron los siguientes factores: intensidad de raleo, posición radial de la muestra, tipo de leño, interacción entre los factores, también se emplearon las siguientes covariables: diámetro a la altura de pecho (dap), altura hasta la base de copa viva (hbcv), longitud de copa viva (lcv). La covariable diámetro a la altura de pecho reemplazó al factor estrato, dado que estrato fue definido en base al diámetro. Para la longitud de traqueidas se determinó que existe variación significativa entre árboles (correlación de 0,44 con p-valor significativo igual a 0,03 ), por lo que se tomó al árbol como fuente adicional de variación. Las variables no significativas fueron suprimidas del análisis.

Los análisis estadísticos se realizaron utilizando el programa Infostat (Di Rienzo et al. 2013), con un nivel de confianza del 95\%.

\section{RESULTADOS Y DISCUSIÓN}

\section{Características de los árboles muestreados}

En la tabla 1 se presentan los resultados de los valores medios determinados para el diámetro a la altura de pecho, altura total, diámetro en la base de la copa viva y altura a la base de la copa viva de los árboles muestreados, los coeficientes de variación y la comparación de medias de los 4 tratamientos, empleando el procedimiento basado en conglomerados y comparación de medias DGC (Di Rienzo et al. 2002).

Tabla 1. Resumen de parámetros dendrométricos del material muestreado según la intensidad de raleo.

\begin{tabular}{|c|c|c|c|c|c|c|c|c|c|}
\hline \multirow[t]{2}{*}{$\begin{array}{c}\text { Intensidad de } \\
\text { raleo }\end{array}$} & \multicolumn{2}{|c|}{ DAP } & \multicolumn{2}{|c|}{$\mathrm{h}$} & \multicolumn{2}{|c|}{ dbcv } & \multicolumn{2}{|c|}{ hbcv } & \multirow[t]{2}{*}{$\mathrm{N}$} \\
\hline & $\begin{array}{l}\text { media } \\
(\mathrm{cm})\end{array}$ & C.V. & $\begin{array}{l}\text { media } \\
(\mathrm{h})\end{array}$ & C.V. & $\begin{array}{l}\text { media } \\
(\mathrm{cm})\end{array}$ & C.V. & $\begin{array}{l}\text { media } \\
(\mathrm{m})\end{array}$ & C.V. & \\
\hline T0:0\% & $26,36 \mathrm{a}$ & $23,1 \%$ & 24,16 a & $9,6 \%$ & $14,95 \mathrm{a}$ & $42,9 \%$ & $16,31 \mathrm{a}$ & $4,6 \%$ & 6 \\
\hline T1:50\% & $29,37 \mathrm{a}$ & $37,1 \%$ & $24,92 \mathrm{a}$ & $14,6 \%$ & $17,17 \mathrm{a}$ & $46,0 \%$ & $15,60 \mathrm{a}$ & $23,4 \%$ & 6 \\
\hline T2:75\% & 34,14 a & $24,8 \%$ & $26,13 \mathrm{a}$ & $15,6 \%$ & $22,25 \mathrm{a}$ & $29,2 \%$ & $14,65 \mathrm{a}$ & $14,2 \%$ & 6 \\
\hline $\mathrm{T} 3: 87,5 \%$ & $43,64 \mathrm{~b}$ & $8,1 \%$ & $25,52 \mathrm{a}$ & $10 \%$ & $29,78 \mathrm{~b}$ & $8,3 \%$ & $11 \mathrm{~b}$ & $32,2 \%$ & 6 \\
\hline Media gral. & 33,38 & $29,5 \%$ & 25,18 & $12,3 \%$ & 21,03 & $38,9 \%$ & 14,39 & $23,1 \%$ & 24 \\
\hline
\end{tabular}

Los valores de diámetro a la altura de pecho y diámetro en la base de la copa viva aumentaron con la intensidad de raleo, resultados similares fueron encontrados por Bravo et al. (2012). Los árboles del tratamiento sin raleo, con mayor número de individuos por hectárea, tuvieron menor crecimiento en diámetro, probablemente debido a la competencia por agua, luz y nutrientes. El raleo favoreció al crecimiento radial, siendo más notable en el de mayor intensidad. En el tratamiento con raleo más intenso los valores de diámetros fueron más homogéneos, presentaron menor dispersión (coeficiente de variación), mientras que el tratamiento con $50 \%$ de raleo presentó mayor dispersión en diámetros. 
La altura hasta la base de copa viva (largo de fuste) disminuye con la intensidad de raleo. Para las variables dap, dbcv y hbcv, el T3 fue estadísticamente diferente a los demás tratamientos. Los valores de altura total se mantuvieron constantes para los cuatro tratamientos.

\section{Ángulo microfibrilar}

El ángulo microfibrilar aumentó con la intensidad de raleo. Los menores valores de AMF se lograron con los tratamientos $0 \%$ y $50 \%$ de raleo $\left(30,8^{\circ}\right)$, seguidos por el correspondiente a $75 \%$ de raleo con $32^{\circ}$. Con el tratamiento de $87,5 \%$ de raleo de la densidad original se obtuvo mayor ángulo microfibrilar, de $34^{\circ}$ (Figura 2). Los valores encontrados son inferiores a los reportados por Huang et al. (1998) para Pinus taeda.

El valor medio del AMF para todas las intensidades de raleo, decreció en dirección radial de la médula hacia la corteza, con valores medios de $34^{\circ}, 33^{\circ}$ y $29^{\circ}$ para las posiciones radiales A, B y C, representando una disminución del 5\% de A a B y del 15\% de A a C (Figura 2).

La disminución radial en el valor del AMF también fue señalada por otros autores (Megraw 1985, Bendtsen y Senft 1986, Donaldson 1992, Walker y Butterfield, 1995, Huang et al. 1998, Saranpää et al. 1998, Herman et al. 1999, Yamashita et al. 2000, Larson et al. 2001, Jones et al. 2005, Zhang et al. 2007, Yin et al. 2011, Winck et al. 2013), asociando claramente valores más elevados de ángulo microfibrilar a la presencia de madera juvenil cercana a la médula.
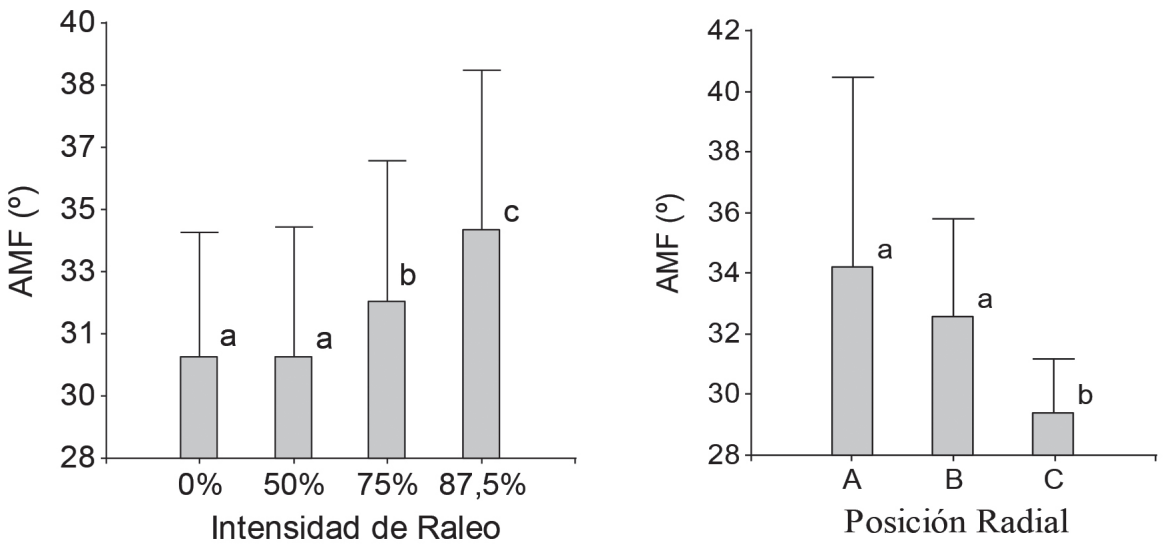

Figura 2. Media del AMF para los tratamientos de raleo y de la posición radial de la muestra para los cuatro tratamientos.

Cave y Walker (1994) indicaron que el MFA para Pinus taeda disminuye a partir de la primera hilera de célula de madera temprana hasta la última hilera de célula de la madera tardía, mostrando los mayores valores cerca de la médula y disminuyendo hacia la corteza, con una rápida variación inicial, con una disminución gradual y finalmente más lenta hasta que se estabiliza. El crecimiento lento (cerca de la corteza) genera fibras de estructura más compacta y menor que en madera temprana, lo que resulta en valores de AMF más bajos (Jordan et al. 2006). 
Winck et al. (2012) determinaron valores medios de AMF que variaron de médula a corteza de $56^{\circ}$ a $42^{\circ}$, lo que representa una disminución del $25 \%$ en el caso de Pinus taeda de 15 años de edad proveniente de un sistema silvopastoril. También Yin et al. (2011) reportaron una disminución radial de $30 \%$ en el valor de AMF para Pinus taeda de 22 años de edad. Mientras que Jordan et al. (2006) registraron una disminución de $18 \%$ en el valor del AMF en árboles de 20 a 27 años en diferentes zonas geográficas. Valores muy similares fueron obtenidos por Isik et al. (2008), lo que estaría indicando que para una mayor edad del árbol, la variación radial del AMF sería menor.

Los valores medios de AMF registrados para las traqueidas correspondiente al leño temprano y tardío fueron de $42^{\circ}$ y $22^{\circ}$ grados respectivamente. El valor del AMF para el leño temprano prácticamente duplica al valor del AMF encontrado para el leño tardío.

Las tablas 2 y 3 muestran los grupos homogéneos determinados para las interacciones entre intensidad de raleo por posición radial ( $\mathrm{p}$-valor $=0,0001)$ e intensidad de raleo por tipo de leño $(\mathrm{p}$-valor $=0,0001)$ para la variable AMF.

Tabla 2. Medias de AMF para intensidad de raleo por posición radial.

\begin{tabular}{cccc}
\hline Intensidad de raleo & Posición radial & AMF medio & Grupo \\
\hline $87,5 \%$ & $\mathrm{~A}$ & 36,02 & $\mathrm{a}$ \\
$75 \%$ & $\mathrm{~A}$ & 35,28 & $\mathrm{a}$ \\
$87,5 \%$ & $\mathrm{~B}$ & 34,41 & $\mathrm{a}$ \\
$0 \%$ & $\mathrm{~A}$ & 33,02 & $\mathrm{~b}$ \\
$50 \%$ & $\mathrm{~A}$ & 32,52 & $\mathrm{~b}$ \\
$87,5 \%$ & $\mathrm{C}$ & 32,47 & $\mathrm{~b}$ \\
$75 \%$ & $\mathrm{~B}$ & 32,20 & $\mathrm{~b}$ \\
$50 \%$ & $\mathrm{~B}$ & 31,88 & $\mathrm{~b}$ \\
$0 \%$ & $\mathrm{~B}$ & 31,83 & $\mathrm{~b}$ \\
$75 \%$ & $\mathrm{C}$ & 29,50 & $\mathrm{c}$ \\
$50 \%$ & $\mathrm{C}$ & 28,00 & $\mathrm{~d}$ \\
$0 \%$ & $\mathrm{C}$ & 27,53 & $\mathrm{~d}$ \\
\hline Los valores medios señalados con letras iguales no difieren estadísticamente entre sí, según test de DGC al $95 \%$ de probabilidad.
\end{tabular}

Los valores medios señalados con letras iguales no difieren estadísticamente entre sí, según test de DGC al $95 \%$ de probabilidad.

Los valores de AMF son menores en la madera más cercana a la corteza, como así también, en la madera con mayor porcentaje de leño tardío. 
Tabla 3. Medias de AMF para intensidad de raleo por tipo de leño.

\begin{tabular}{cccc}
\hline Intensidad de raleo & Tipo de leño & AMF medio & Grupo \\
\hline $87,5 \%$ & temprano & 44,75 & $\mathrm{a}$ \\
$0 \%$ & temprano & 42,42 & $\mathrm{~b}$ \\
$75 \%$ & temprano & 40,80 & $\mathrm{c}$ \\
$50 \%$ & temprano & 40,19 & $\mathrm{c}$ \\
$75 \%$ & tardío & 23,85 & $\mathrm{~d}$ \\
$87,5 \%$ & tardío & 23,85 & $\mathrm{~d}$ \\
$50 \%$ & tardío & 21,41 & $\mathrm{e}$ \\
$0 \%$ & tardío & 19,17 & $\mathrm{f}$ \\
\hline
\end{tabular}

No se encontró interacción entre los factores posición radial y tipo de leño ( $p$-valor=0,4716). El leño tardío presentó menores valores de AMF independientemente de la posición radial (tabla 4). El tipo de leño es una fuente de variación importante para AMF. Las traqueidas provenientes del leño tardío presentaron menor AMF que las traqueidas del leño temprano, independientemente de la posición radial, indicando que el tipo de leño tiene mayor influencia que la posición radial sobre el valor de AMF.

Tabla 4. Medias de AMF para posición radial por tipo de leño.

\begin{tabular}{cccc}
\hline Posición radial & Tipo de leño & AMF medio & Grupo \\
\hline A & temprano & 44,67 & $\mathrm{a}$ \\
B & temprano & 42,47 & $\mathrm{~b}$ \\
C & temprano & 38,99 & $\mathrm{c}$ \\
A & tardío & 23,75 & $\mathrm{~d}$ \\
B & tardío & 22,69 & $\mathrm{~d}$ \\
C & tardío & 19,76 & $\mathrm{e}$ \\
\hline Los valores medios señalados con letras iguales no difieren estadísticamente entre sí, según test de DGC al 95\% de probabilidad.
\end{tabular}

Si bien sería de esperar menores valores de ángulos microfibrilares para la edad de 20 años, se debe tener en cuenta que la técnica de medición empleada sobreestima los valores para las traqueidas de madera temprana (Huang et al. 1998).

\section{Espesor de pared celular}

En la figura 3 se muestra la variación media del espesor de pared según el tratamiento de raleo aplicado y según la posición radial para los cuatro tratamientos.
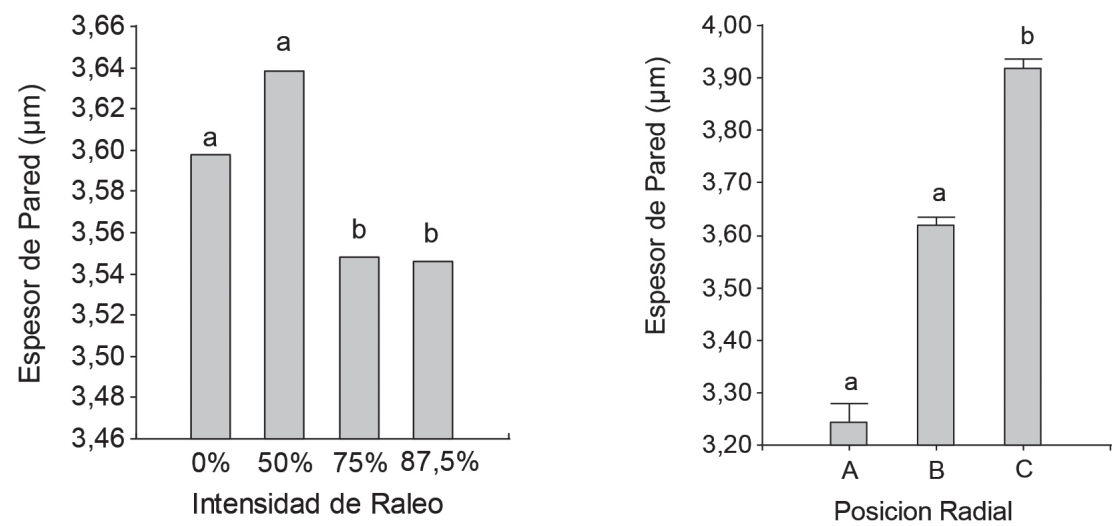

Figura 3. Media del espesor de pared celular para la intensidad de raleo y la posición radial para los cuatro tratamientos. 
El espesor de pared fue mayor en los tratamientos con $0 \%$ y $50 \%$ de raleo, con valores de 3,60 $\mu \mathrm{m}$ y $3,64 \mu \mathrm{m}$ respectivamente, y fueron algo menores aunque iguales para las intensidades de raleo de $75 \%$ y $87,5 \%(3,55 \mu \mathrm{m})$. Se notó una disminución en el espesor de pared celular al aumentar la intensidad de raleo.

Con respecto a la posición radial, aumentó desde la medula hacia la corteza, con valores medios de $3,24 \mu \mathrm{m}, 3,62 \mu \mathrm{m}$ y $3,92 \mu \mathrm{m}$ para las posiciones radiales A, B y C respectivamente, para todas las intensidades de raleo. La posición radial C, presentó el mayor espesor de pared celular, siendo estadísticamente superior a los valores de la posición radial A y B.

Como se esperaba, dado que el leño tardío se caracteriza por presentar traqueidas más alargadas y con mayor espesor de pared, los valores medios de espesor de pared obtenidos para las traqueidas de leño temprano y tardío fueron estadísticamente diferentes, registrándose valores medios de 3,04 $\mu \mathrm{m}$ y $4,22 \mu \mathrm{m}$ respectivamente.

Los tratamientos de $0 \%$ y $50 \%$ de raleo produjeron valores similares de AMF y espesor de pared. En los tratamientos con $75 \%$ y $87,5 \%$ de raleo estas propiedades fueron negativamente afectadas, el espesor de pared celular disminuyó y el AMF aumentó.

En la figura 4 se presenta la interacción para intensidad de raleo y posición radial para el espesor de pared celular.

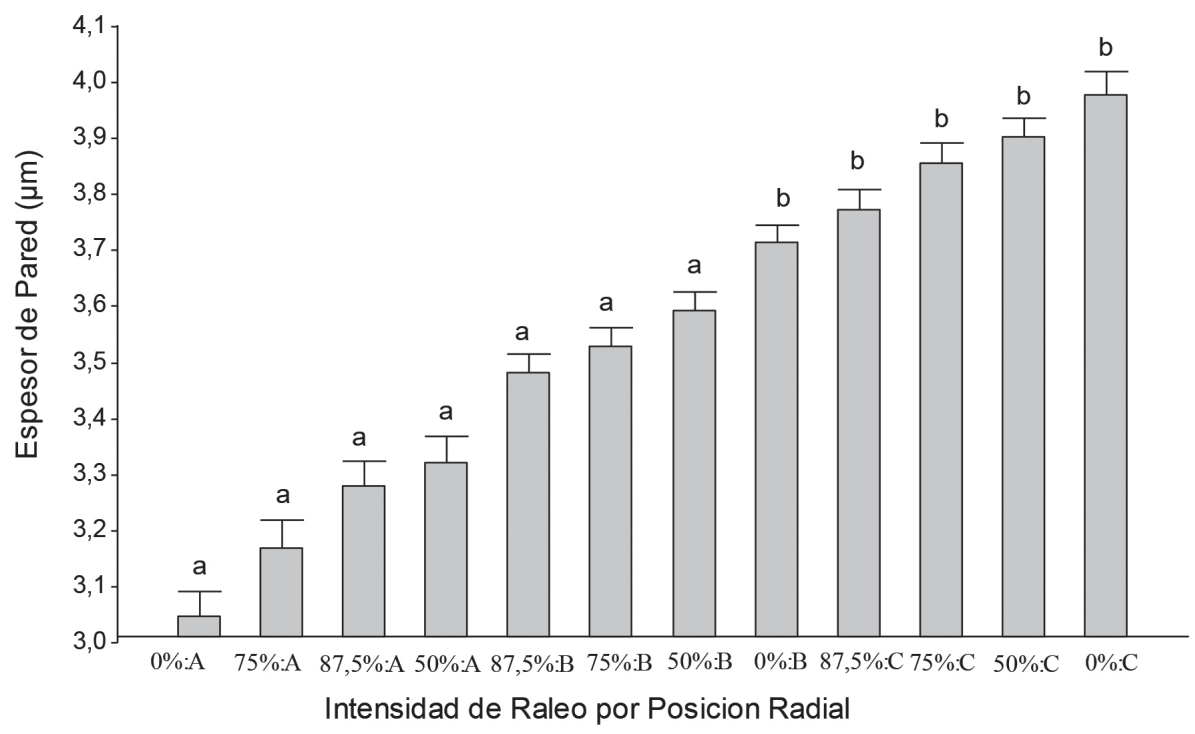

Figura 4. Variación del espesor de pared con respecto a la intensidad de raleo y posición radial. Donde $0 \%, 50 \%, 75 \%$ y $87,5 \%$ es la intensidad de raleo. A, B y C corresponden a la posición radial interna, media y externa respectivamente.

La interacción entre intensidad de raleo y posición radial fue significativa ( $p$-valor $=0,0001)$. Se determinaron dos grupos estadísticamente diferentes. El grupo "b" que presentó mayor espesor de pared, formado por la posición radial C para todos los tratamientos, con el orden T0, T1, T2 y T3 y la posición radial B del T0. El grupo "a", con menor espesor de pared, quedó conformado por las posiciones radiales B y A para los 4 tratamientos. La posición radial A (cercana a la médula) sin raleo (T0) presentó los menores espesores de pared celular. 
La interacción entre intensidad de raleo y tipo de leño fue significativas ( $p$-valor $=0,0006$ ). Se evidenció que para los 4 tratamientos el espesor de pared de las traqueidas de leño tardío es muy superior al de las traqueidas de leño temprano. El valor medio del espesor de pared para los 4 tratamientos fue un $28 \%$ mayor en las traqueidas del leño tardío.

La figura 5 muestra la interacción entre posición radial y tipo de leño para espesor de pared celular.

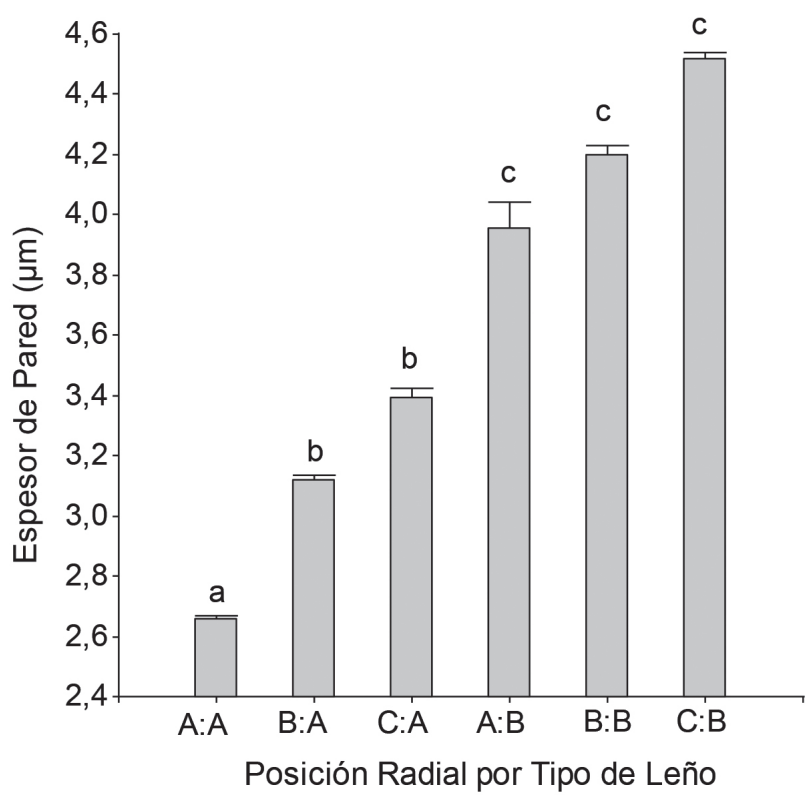

Figura 5. Variación del espesor de pared celular con respecto a la posición radial y el tipo de leño. Donde A, B y C corresponden a la posición radial interna, media y externa respectivamente.

Tem=leño temprano, Tar=leño tardío.

La interacción entre posición radial y tipo de leño resultó significativa ( $\mathrm{p}$-valor $=0,0001)$. El espesor de pared aumentó radialmente, tanto en leño temprano como tardío, identificándose 3 grupos. El espesor de pared del leño tardío de la posición radial A es mayor que el del leño temprano de la posición radial C. El menor valor se registró para el leño temprano de la posición radial A. Esto indica que el espesor de pared celular de las traqueidas varía en mayor medida con el tipo de leño, que con la posición radial de donde se extrajo la muestra. 


\section{Longitud de traqueidas}

En la figura 6 se muestra la variación media en la longitud de traqueidas en función de la intensidad de raleo y la posición radial para los cuatro tratamientos.
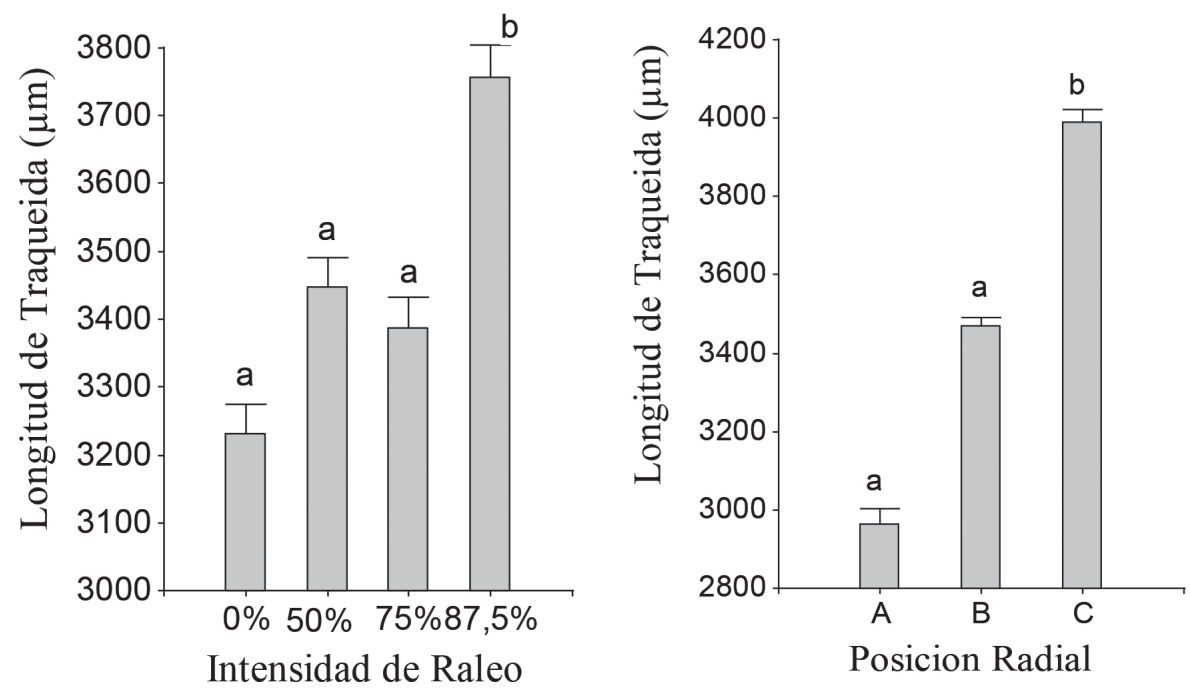

Figura 6. Longitud media de traqueidas para la intensidad de raleo y la posición radial de los cuatro tratamientos.

La intensidad de raleo provocó una alteración en la longitud de traqueidas, pero sin marcar una tendencia clara y sistemática positiva o negativa en función al espacio vital asignado para cada árbol debido al tratamiento aplicado. Los tratamientos T0, T1 y T2 alcanzaron longitudes estadísticamente iguales de $3232 \mu \mathrm{m}, 3447 \mu \mathrm{m}$ y $3388 \mu \mathrm{m}$ respectivamente. El tratamiento con 87,5\% de raleo presentó una longitud media de traqueidas de $3756 \mu \mathrm{m}$, un $11 \%$ mayor que la media de los tratamientos T0, T1 y T2 (Figura 6). Jordan el al. (2006) indican que el crecimiento lento podría producir fibras de estructura más compacta, lo que podría explicar el comportamiento encontrado, que en tratamientos más densos la longitud de traqueidas fue menor.

La mayor longitud de traqueidas del tratamiento con $87,5 \%$ de raleo probablemente se deba a la mayor proporción de copa viva, dado que según Jyske (2008) la longitud de traqueidas está ligada a la proporción de brotes vivos.

Por otra parte, los árboles que tuvieron raleo más intenso crecieron en condiciones más favorables, con un mayor crecimiento radial del árbol y con mayor longitud de copa verde. Esto coincide con lo reportado por Jyske (2008), quien encontró que los árboles dominantes presentaron mayor longitud de traqueidas que los árboles suprimidos, en este caso, los árboles dominantes fueron seleccionados en función al diámetro, y en este estudio, el tratamiento que recibió $87,5 \%$ de raleo tuvieron árboles de mayores diámetros, respecto a los demás tratamientos. Trevisan et al. (2013) también determinaron que los árboles dominantes de Eucalyptus grandis presentaron mayor longitud de fibras $(4,2 \%)$ que los árboles medios. Estos mismos autores, establecieron que la intensidad de raleo provocó alteraciones poco claras en la longitud de fibras de los árboles dominantes, no encontraron un patrón determinado, mientras que para los árboles del estrato medio, la tendencia fue clara, la longitud de traqueidas aumentó con la intensidad de raleo. Sin embargo otros autores (Helander 1933, Dinwoodie 1961, Herman et al. 1998, Mäkinen y Isomäki 2002, Lundgren 2004), han reportado resultados contradictorios sobre la relación entre la tasa de crecimiento radial y la longitud de traqueidas, donde la longitud de traqueidas disminuyó con la intensidad de raleo. 
La variación radial de la longitud de traqueidas fue ascendente, independientemente de la intensidad de raleo, con valores medios de $2966 \mu \mathrm{m}, 3471 \mu \mathrm{m}$ y $3992 \mu \mathrm{m}$ para las posiciones radiales A, B y C respectivamente (Figura 6). La longitud de traqueidas aumentó un $15 \%$ de la posición radial A a B y un $26 \%$ de la posición A a C. El incremento de B a C fue de $11 \%$, mostrando una leve tendencia a la estabilización de la longitud de las traqueidas, característica de la madera madura.

El valor promedio de la longitud de traqueidas determinado en este trabajo para la madera interna (posición radial A y B) y externa (posición radial C) para todas las intensidades de raleo fue de 3218 um y $3992 \mu \mathrm{m}$ respectivamente, similares a las encontradas por Bolzón (1993) para esta misma especie a los 30 años de edad (madera juvenil: $3415 \mu \mathrm{m}$, madera madura $4002 \mu \mathrm{m}$ ), pero más cortas que las longitudes hallada por Ballarín y Palma (2003) a los 37 años de edad (madera juvenil: $4200 \mu \mathrm{m}$, madura $5322 \mu \mathrm{m})$. Esto se debe probablemente a las diferencias de edad.

El leño tardío presentó traqueidas 34\% más larga que las traqueidas del leño temprano (4257 $\mu \mathrm{m}$ y $2797 \mu$ m respectivamente).

La interacción intensidad de raleo y posición radial fue significativa ( $\mathrm{p}$-valor $=0,0001)$. Existen dos grupos de medias homogéneos. El grupo que menor longitud de traqueidas presentó estuvo compuesto por los tratamientos T0, T1 T2 en la posición radial A.

La interacción intensidad de raleo y tipo de leño, no fue significativa ( $p$-valor $=0,0518$ ). El leño tardío presentó mayor longitud de traqueidas en los 4 tratamientos. Los tratamientos que alcanzaron mayor longitud de traqueidas fueron $\mathrm{T} 3>\mathrm{T} 1>\mathrm{T} 2>\mathrm{T} 0$ para leño temprano y tardío.

La longitud media de traqueidas del leño tardío (de las 3 posiciones radiales) fue un $33 \%$ superior a la longitud media de traqueidas del leño temprano (de las 3 posiciones radiales). La longitud de traqueidas varía en mayor medida con el tipo de leño, que con la posición radial de la muestra.

La interacción entre los factores posición radial y tipo de leño resultó significativa ( $\mathrm{p}$-valor $=0,0001$ ). En la tabla 5 se muestra la interacción entre la posición radial y el tipo de leño para la longitud de traqueidas.

Tabla 5. Variación de la longitud de traqueidas para la posición radial por tipo de leño.

\begin{tabular}{cccc}
\hline Posición radial & Tipo de leño & Long media traqueidas $(\mu \mathrm{m})$ & Grupo \\
\hline C & tardío & 4715 & $\mathrm{a}$ \\
A & tardío & 4056 & $\mathrm{a}$ \\
B & tardío & 4033 & $\mathrm{a}$ \\
C & temprano & 3379 & $\mathrm{~b}$ \\
B & temprano & 2987 & $\mathrm{~b}$ \\
A & temprano & 2168 & $\mathrm{c}$ \\
\hline
\end{tabular}

Los valores medios señalados con letras iguales no difieren estadísticamente entre sí, según test de DGC al 95\% de probabilidad.

La variación radial en las características de los elementos anatómicos (espesor de pared celular, longitud de traqueidas y ángulo microfibrilar), como así también de otras propiedades, se debe a la presencia de una zona de madera madura cercana a la corteza y una zona de madera juvenil cercana a la médula, que presenta entre otras características, traqueidas más cortas, paredes celulares más delgadas y mayor ángulo microfibrilar en la capa S2 (Bendtsen 1978, Senft et al. 1985 y Burdon et al. 2004), lo que repercute directamente en la calidad de la madera (Trevisan et al. 2013). 


\section{CONCLUSIONES}

Las intensidades de raleo estudiadas produjeron variaciones significativas en las propiedades anatómicas de la madera de Pinus taeda. El ángulo microfibrilar y la longitud de traqueidas aumentaron, mientras que el espesor de pared disminuyó con la intensidad de raleo.

La aplicación de un raleo del 50\% de la densidad original de la plantación favoreció la obtención de madera con mayor espesor de pared celular y menor ángulo microfibrilar. Por el contrario, el tratamiento con $87,5 \%$ de raleo generó un aumento en el ángulo microfibrilar y una disminución en el espesor de pared.

El raleo provocó una alteración en la longitud de traqueidas, pero sin marcar una tendencia clara y sistemática positiva o negativa, aunque en el tratamiento con mayor intensidad, las traqueidas fueron más largas.

El ángulo microfibrilar disminuyó en el sentido radial de médula a corteza, mientras que el espesor de pared y la longitud de traqueidas aumentaron en el sentido radial independientemente del tratamiento de raleo aplicado.

La madera de leño tardío presentó mayor longitud de traqueidas, mayor espesor de pared y menor ángulo microfibrilar que la madera de leño temprano.

En la posición radial inmediatamente cerca de la médula y al 50\% del radio, se registraron valores estadísticamente iguales para las variables espesor de pared, longitud de traqueidas y ángulo microfibrilar, indicando que a los 9 años, el Pinus taeda aún está constituido totalmente por leño juvenil (la posición ubicada al 50\% de la distancia del radio abarcó como máximo al noveno anillo de crecimiento).

\section{AGRADECIMIENTOS}

Al Ministerio de Agricultura, Ganadería y Pesca, Componente Plantaciones Forestales SustentablesBIRF 7520-AR por el financiamiento brindado a través del PIA 10003. A la Empresa APSA S.A. que facilitó el sitio donde se instaló oportunamente el ensayo de raleo, de donde se obtuvieron las muestras para este estudio. 


\section{REFERENCIAS}

Ballarin, A.W.; Palma, H.A.L. 2003. Propriedades de resistência e rigidez da madeira juvenil e adulta de Pinus taeda L. Revista Árvore 27 (3): 371-380.

Bendtsen, B.A. 1978. Properties of wood from improved and intensively managed trees. Forest Products Journal 28 (10):61-72.

Bendtsen, B.A.; Senft, J. 1986. Mechanical and anatomical properties in individual growth rings of plantation grown eastern cotton wood and loblolly pine. Wood and Fiber Science 18(1): 23-38.

Bolsón de Muñiz, G. 1993. Caracterización e desenvolvimento de modelos para estimar as propiedades e o comportamento na secagem da madeira de Pinus elliotti Engelm. E Pinus taeda L. Tese doutoral em Ciências Florestais. Universidade Federal do Paraná. Curitiba, Brasil.

Bravo, S.D.; Espinosa, M.; Valenzuela, L.; Cancino, J.; Lasserre, J.P. 2012. Efecto del raleo en el crecimiento y algunas propiedades de la madera de Eucalyptus nitens en una plantación de 15 años. Maderas. Ciencia y tecnología 14(3):373-388.

Burdon, R.D.; Kibblewhite, R.P.; Walker, J.C.F.; Megraw, R.A. 2004. Juvenile versus mature wood: a new concept, orthogonal to corewood versus outerwood, with special reference to Pinus radiata and P. taeda. For Sci 50: 399-415.

Cave, I.D., Walker, J.C.F. 1994. Stiffness of wood in fast-grown plantation softwoods: The influence of microfibril angle. Forest Prod J 44(5):43-48.

Di Rienzo, J.A.; Guzmán A.W.; Casanoves F. A. 2002. Multiple Comparisons Method based on the Distribution of the Root Node Distance of a Binary Tree. Journal of Agricultural, Biological, and Environment Statistics 7(2): 1-14.

Di Rienzo J.A.; Casanoves F.; Balzarini M.G.; Gonzalez L.; Tablada, M.; Robledo C.W. InfoStat versión 2013. Grupo InfoStat, FCA, Universidad Nacional de Córdoba, Argentina.

Dinwoodie, J.M. 1961. Tracheid and fibre length in timber. A review of literature. Forestry 34:125144.

Donaldson, L.A. 1992. Within and between tree variation in microfibril angle in Pinus radiate. Journal of Forestry Science 22(1):77-86.

Donaldson, L.A. 1996. Effect of physiological age and site on microfibril angle in Pinus radiate. IAWA Journal 17(4):421-429.

Helander, A.B. 1933. Variations in tracheid length of pine and spruce. Foundation for forest research of Finland. No. 14. 75 p.

Hein, P.R.G.; Lima, J.T. 2012. Relationships between microfibril angle, modulus ofvelasticity and compressive strength in Eucalyptus Wood. Maderas. Ciencia y tecnología 14(3): 267-274.

Hein, P.R.G.; Silva, J.R.M.; Brancheriau, L. 2013. Correlations among microfibril angle, density, modulus of elasticity, modulus of rupture and shrinkage in 6-year-old Eucalyptus urophylla $\times$ E. grandis. Maderas. Ciencia y tecnología 15(2): 171-182. 
Herman, M., Dutilleul, P.; Avella-Shaw, T. 1998a. Growth rate effects on temporal trajectories of ring width, wood density, and mean tracheid length in Norway spruce (Picea abies (L.) Karst.). Wood Fiber Sci 30(1): 6-17.

Herman, M.; Dutilleul, P.; Avella-Shaw, T. 1999. Growth rate effects on intra-ring and inter-ring trajectories of microfibril angle in Norway spruce (Picea abies). IAWA Journal 20: 3-21.

Huang, C.L.; Kutscha, N.P.; Leaf, G.J.; Megraw, R.A. 1998. Comparison of microfibril angle measurement techniques. In: B.G. Butterfield (ed.), Microfibril angle in wood: 177-205. IAWA and IUFRO, Christchurch, New Zealand.

INTA. 1990. Atlas de Suelo de la República Argentina. Provincia de Misiones, tomo II, 111-154.

Isik, F.; Gumpertz, M.; Li, B.; Goldfarb, B.; Sun, X. 2008. Analysis of cellulose microfibril angle using a linear mixed model in Pinus taeda clones. Can J For Res 38: 1676-1689.

Jones, P.D.; Schimleck, L.R.; Peter, G.F.; Daniels, R.F.; Clark, A.III. 2005. Nondestructive estimation of Pinus taeda L. wood properties for samples from a wide range of sites in Georgia. Can $J$ For Res 35: 85-92.

Jordan, L.; Hall, D.B.; Clark, A. III; Daniels, R.F. 2006. Variation in loblolly pine cross-sectional microfibril angle with tree height and physiographic region. Wood and Fiber Science 38(3): 390-398.

Jyske, T. 2008. The effects of thinning and fertilisation on wood and tracheid properties of Norway spruce (Picea abies) the results of long-term experiments. Dissertationes Forestales 55. Department of Forest Resource Management. Faculty of Agriculture and Forestry. University of Helsinki.

Köhl, M.; Magnussen, S.; Marchetti, M. 2006. Sampling Methods; Remote Sensing and GIS. Multiresource Forest Inventory. Springer. Berlin, Heidelberg. Serie Tropical Forestry p.17-69.

Larson, P.R.; Kretschmann, D.E.; Clark, A III.; Isebrands, J.G. 2001. Formation and properties of juvenile wood in southern pines: A synopsis. Gen. Tech. Rep. FPL-GTR-129. USDA, Forest Serv., Forest Prod. Lab, Madison, WI. 42p.

Lundgren, C. 2004. Cell wall thickness and tangential and radial cell diameter of fertilized and irrigated Norway spruce. Silva Fenn 38(1): 95-106.

Macdonald, E.; Hubert, J. 2002. A review of the effects of silviculture on timber quality of Sitka spruce. Forestry 75 (2):107-138.

Mäkinen, H.; Isomäki, A. 2004a. Thinning intensity and growth of Norway spruce stands in Finland. Forestry 77(4): 349-364.

Megraw, R.A. 1985. Wood quality factors in loblolly pine. The influence of tree age, position in tree, and cultural practice on wood specific gravity, fiber length, and fibril angle. TAPPI PRESS. Atlanta, GA. $88 \mathrm{p}$.

Núñez, C.E.; Pavlik, C.A. 1999. Disgregado de tejidos leñosos por el método clorito-ácido acéticocarbonato. Evaluación del daño producido a las fibras. Rev Cienc Tecnol 2(2):33-37.

Richter, H.G.; Grosser, D.; Heinz, I.; Gasson, P.E. (eds). 2004. IAWA list of microscopic features for softwood identification. IAWA Journal 25(1): 1-70. 
Saranpää, P.; Serimaa, R.; Andersson, S.; Pessonen, E.; Suni, T.; Paakkari, T. 1998. Variation of microfibril angle Norway spruce (Picea abies (L.) Karst.) and Scots pine (Pinus sylvestris L.)-comparing X-ray diffraction and optical methods. In: B.G. Butterfield (ed.), Microfibril angle in wood. p: 240-252.

Sarén, M.; Serimaa, R.; Andersson, S.; Saranpää, P.; Keckes, J.; Fratzl, P. 2004. Efect of growth rate on mean microfibril angle and cross-sectional shape of tracheids of Norway spruce. Trees 18:354-362.

Senft, J.F.; Bendtsen, B.A.; Galligan, W.L. 1985. Weak wood. Journal of Forestry (83): 476-485.

Servicio Meteorológico Nacional. 2008. Datos Estadísticos. Período 1981-1990. Ciudades de Iguazú y Posadas.

Soil Survey Staff. 1975. Soil Taxonomy. United States Department of Agriculture Handbook, Washington, D.C. 436p.

Trevisan, R.; Souza, J.T.De; Denardi, L.; Haselein, C.R.; Santini, E.J. 2013. Efeito do desbaste no comprimento das fibras da madeira de Eucalyptus grandis W. Hill ex Maiden. Ciência Florestal 23(2): 461-473.

Walker, J.C.F.; Butterfield, B.G. 1995. The importance of microfibril angle for the processing industries. New Zealand Forestry 4(4):34-40.

Wang, H.H.; Drummond, J.G.; Reath, S.M.; Hunt, K.; Watson, P.A. 2001. An improved fibril angle measurement method for wood fibres. Wood Sci Technol 34: 493-503.

Wilcox, C.D.; Dove, S.B.; McDavid, W.D.; Greer, D.B. 2002. UTHSCSA Image Tool.

Winck, R.A.; Fassola, H.E.; Tomazello, M.F.; Area, M.C. 2012. Caso de estudio: Angulo microfibrilar y su relación con la densidad básica de la madera de Pinus taeda L. con manejo silvopastoril. $\mathrm{VII}^{\circ}$ Edición Congreso Iberoamericano de Investigación Celulosa y Papel.

Winck, R.A.; Fassola, H.E.; Tomazello, M.F.; Area, M.C. 2013. Case study: Microfibril angle and its relationship with basic density in Pinus taeda L. Wood from silvopastoral systems. O Papel 74 (5): 55-61.

Yamashita, K.; Hirakawa, Y.; Fujisawa, Y.; Nakada, R. 2000. Effects of microfibril angle and density on variation of modulus of elasticity of Sugi (Cryptomeria japonica) logs among eighteen cultivars. Mokuzai Gakkaishi 46: 510-522.

Ying, L.; Kretschmann, D.E.; Bendtsen, B.A. 1994. Longitudinal shrinkage in fast-grown loblolly pine plantation wood. Forest Prod J 44(1):58-62.

Yin, Y.; Bian, M.; Song, K.; Xiao, F.; Xiaomei, J. 2011. Influence of microfibril angle on within tree variations in the mechanical properties of chinese fir (Cunninghamia lanceolata). IAWA Journal 32(4):431-442.

Yin, Y.; Song, K.; Liu, B.; Jiang, X. 2011. Variation of microfibril angle in plantation trees of Cunninghamia lanceolata determined by pit apertures and x-ray diffraction. IAWA Journal 32 (1): 77-87.

Zhang, B.; Fei, B.H.; Yu, Y.; Zhao, R.J. 2007. Microfibril angle variability in Masson pine (Pinus massoniana Lamb.) using X-ray diffraction. Forestry Studies in China 9: 33-38. 